\section{Occurrence of mislabelling in prepared fishery products in Southern Italy}

Giuseppina Tantillo, Patrizia Marchetti, Anna Mottola, Valentina Terio, Marilisa Bottaro, Elisabetta Bonerba, Giancarlo Bozzo, Angela Di Pinto

Department of Veterinary Medicine, University of Bari, Italy

\section{Abstract}

Fish authentication is a major concern not only for the prevention of commercial fraud, but also for the assessment of safety risks deriving from the undeclared introduction of potentially dangerous toxic or allergenic substances or environmentally damaging fish where endangered species are involved. Moreover, food authentication might affect the diet of certain groups of consumers, such as followers of religious practices. Considering the authentication of fish products is one of the key issues in food safety, quality and sustainability, the aim of this work was to investigate the prevalence of mislabelling in sole (Solea solea), plaice (Pleuronectes platessa), Atlantic salmon (Salmo salar), and hake (Merluccius merluccius) fillets from markets and supermarkets located in Apulia (Southern Italy) using DNA barcoding. The results of the molecular investigations reveal that $42 / 98$ (42.8\%) fillet samples were not correctly labelled. In particular, 12/27 (44.4\%) fillets of sole (Solea solea) were identified as belonging to Solea senegalensis. In addition, 13/28 (46.4\%) plaice (Pleuronectes platessa) samples were identified as Pangasius hypophtalmus. All Atlantic salmon (Salmo salar) samples were correctly labelled. Post-sequencing data analysis revealed that $17 / 30 \quad(56.6 \%)$ hake fillets (Merluccius merluccius) were not correctly labelled, of which $8 / 30$ samples identified as Merluccius hubbsi, 5/30 samples as Merluccius products and 4/30 as Merluccius capensis. The study reveals a high occurrence of species mislabelling in the prepared fish fillet products, further evidence of the need for increased traceability and assessment of the authenticity of food products.

\section{Introduction}

The increasing demand for fishery products in general may lead to deliberate adulteration along the food chain, due to the substitution of high-quality species by lower quality counterparts. The authentication of prepared fish products is one of the key issues in food safety, quality and sustainability. Prepared fishery products, i.e. unprocessed fishery products that have undergone an operation affecting their anatomical wholeness are vulnerable to fraudulent labelling due to the economic profits arising from selling cheaper species as high-value ones (Di Pinto et al., 2013). Moreover, different fish species may be similar in taste and texture, which makes it very difficult to identify the species correctly when the fish is delivered without its diagnostic body parts (e.g. skin, entrails, head and fins), or when it is turned into fillets or slices. Food authentication is a major concern not only for the prevention of commercial fraud, but also for the increased awareness among consumers regarding the composition of foods and the need to verify labelling statements (Bottero and Dalmasso, 2011; Armani et al., 2015). In addition, fish substitution or mislabelling may be significant from a sanitary point of view because of potentially dangerous toxic or allergenic substances, or else environmentally damaging where endangered species are involved (Marko et al., 2004; Ward et al., 2008; Wong and Hanner, 2008; Holmes et al., 2009). Moreover, food authentication might affect the diet of certain groups of consumers, such as followers of religious practices (Di Pinto et al., 2015, in press). Considering that seafood mislabelling has been reported throughout the world (Jacquet and Pauly, 2008; Cawthorn et al., 2011; Garcia-Vazquez et al., 2011; Hanner et al., 2011; Cline, 2012; Miller et al., 2012; Di Pinto et al., 2013, 2015) and that the authentication of food components is one of the key issues in food quality and safety, the aim of this study was to investigate the prevalence of mislabelling in sole (Solea solea), plaice (Pleuronectes platessa), Atlantic salmon (Salmo salar) and hake (Merluccius merluccius) fillets from markets and supermarkets located in Apulia (Southern Italy) using DNA barcoding (Hebert et al., 2003).

\section{Materials and Methods}

\section{Sampling}

A total of 98 samples of prepared fresh fillet fish products, including 27 sole (Solea solea), 28 European plaice (Pleuronectes platessa), 13 Atlantic salmon (Salmo salar) and 30 hake (Merluccius merluccius) from fish retail outlets fish retail premises, fish markets, supermarkets and hypermarkets located in Apulia (Southern Italy) were collected and stored at $-20^{\circ} \mathrm{C}$ until processing. According to Council Regulation (EC) No 1379/2013 (European Comission, 2013) applicable from 01/01/2014, consumer labelling requirements (commercial designation, scientific name, production method and geographical area, whether previously frozen) were considered.
Correspondence: Giuseppina Tantillo, Department of Veterinary Medicine, University of Bari Aldo Moro, Strada Prov. le Casamassima km 3,70010 Valenzano (BA), Italy.

Tel: +39.080 .5443853 - Fax: +39.080 .5443855$

E-mail: giuseppina.tantillo@uniba.it

Key words: Mislabelling; Prepared fish products; DNA barcoding; Southern Italy.

Conflict of interest: the authors declare no potential conflict of interest.

Received for publication: 9 June 2015. Revision received: 26 June 2015.

Accepted for publication: 26 June 2015.

This work is licensed under a Creative Commons Attribution 3.0 License (by-nc 3.0).

(C) Copyright G. Tantillo et al., 2015

Licensee PAGEPress, Italy

Italian Journal of Food Safety 2015; 4:5358

doi:10.4081/ijfs.2015.5358

\section{DNA extraction and purification}

Aliquots of each sample (10 mg) were subjected to DNA extraction and purification using the DNeasy Blood \& Tissue Kit (QIAGEN, Hilden, Germany) as reported by Handy et al. (2011). Positive extraction controls were obtained from each specimen of authentic species. A negative extraction control (no added tissue) was included to verify the purity of the extraction reagents. The DNA concentration and purity were established by evaluating the ratio A260nm/A280nm using a Beckman DU640B Spectrophotometer.

\section{Oligonucleotide primers}

The oligonucleotide primers, FISHC01LBC: 5'-TCAACYAAT CAYAAAGATATYGGCAC-3' and FISHCO1HBC: 5'-ACTTCYGGGTGRCCR AARAATCA-3' reported by Handy et al. (2011) and synthesized by EUROFINS GENOMICS Srl (Milan, Italy), were used.

\section{Polymerase chain reaction assay}

The PCR reactions were performed in a final volume of $25 \mu \mathrm{L}$, using $12.5 \mu \mathrm{L}$ of HotStarTaq Master Mix 2X (QIAGEN, Hilden, Germany), containing 2.5 units of HotStarTaq DNA Polymerase, $1.5 \mathrm{mM}$ of $\mathrm{MgCl}_{2}$ and $200 \mu \mathrm{L}$ of each dNTP. Then, $1 \mu \mathrm{M}$ of each oligonucleotide primer and $1 \mu \mathrm{L}(40 \mathrm{ng} / \mathrm{\mu L})$ of DNA were added. The amplification profile involved an initial denaturation step at $95^{\circ} \mathrm{C}$ for $15 \mathrm{~min}$, followed by 30 cycles at $94^{\circ} \mathrm{C}$ for $30 \mathrm{~s}, 50^{\circ} \mathrm{C}$ for $40 \mathrm{~s}$ and $72^{\circ} \mathrm{C}$ for $60 \mathrm{~s}$. The positive and negative controls for the extraction and PCR were included. The PCR reactions were processed in a Mastercycler Personal (Eppendorf, Milan, Italy). All reactions were performed in duplicate. 


\section{Detection of amplified products}

PCR amplified products were analyzed by electrophoresis on $1.5 \%(\mathrm{w} / \mathrm{v})$ agarose NA (Pharmacia, Uppsala, Sweden) gel in 1X TBE buffer containing $0.089 \mathrm{M}$ Tris, $0.089 \mathrm{M}$ boric acid, 0.002 M EDTA, pH 8.0 (USB, Cleveland, OH, USA), and stained with Green Gel Safe 10000X Nucleic Acid Stain $(5 \mu \mathrm{L} / 100 \mathrm{~mL})$ (Fisher Molecular Biology, USA). A Gene Ruler $^{\text {TM }} 100$ bp DNA Ladder Plus (MBI Fermentas, Vilnius, Lithuania) was used as the molecular weight marker. Image acquisition was performed using UVITEC (Eppendorf).

\section{Polymerase chain reaction cleanup}

In order to produce an amplicon free of extra dNTPs and excess primers that might interfere with the sequencing reaction, the PCR products were purified with the QIAquick PCR Purification Kit (QIAGEN, Hilden, Germany).

\section{Cycle sequencing reaction}

Sequencing reactions using were performed as described by Handy et al. (2011) and carried out by EUROFINS GENOMICS Srl (Milan, Italy).

\section{Sequence analysis}

All amplified sequences were compared with sequences available in the Barcode of Life Data System (BOLD) and GenBank databases using Geneious Pro v5.4 (Drummond et al., 2011). The bidirectional sequences with $98 \%$ HQ (98\% high-quality bases) were compared with sequences from the BOLD and GenBank databases.

\section{Results}

The labels of only $37 / 98$ fish fillet samples provided comprehensive information relating to the commercial designation, scientific name, geographical area, production method and whether they were previously frozen, according to the art. 35 of the Council Regulation (EC) n.1379/2013 (European Commission, 2013). The labelling of other samples was not compliant with European legislation. In particular, the scientific name was missing in 49/98 samples, the geographical area was omitted in $39 / 98$, the commercial designation and the production method were reported in all samples.

The results of the molecular investigations reveal a high occurrence of incorrect species declaration in prepared fillet products (Table 1). Considering the Decree of the Italian Ministry of Agricultural, Food and Forestry Policies (MiPAAF) dated 31 January 2008, which reports the Italian name for fish species of commercial interest, the commercial and/or scientific name declared failed to match the species identified in 42/98 (42.8\%) samples (Table 1). In particular, DNA of sufficient yield and quality was isolated and purified from all samples. The sequences obtained from the samples and compared against the BOLD and GenBank databases gave successful matches, varying from $98 \%$ to $100 \%$ pair wise sequence identity. Post-sequencing data analysis revealed that $42 / 98(42.8 \%)$ fillet samples were not correctly labelled (Table 1). In particular, 12/27 (44.4\%) fillets of sole (Solea solea) were identified as belonging to Solea senegalensis. In addition, 13/28 (46.4\%) plaice (Pleuronectes platessa) samples were identified as Pangasius hypophtalmus. All Atlantic salmon (Salmo salar) samples were correctly labelled. Postsequencing data analysis revealed that $17 / 30$ (56.6\%) hake fillets (Merluccius merluccius) were not correctly labelled, of which $8 / 30$ samples identified as Merluccius hubbsi, 5/30 samples as Merluccius products and 4/30 as Merluccius capensis.

\section{Discussion}

In order to ensure high levels of safety, quality and transparency in seafood products, European Union food law implements the principle of quality management and processoriented controls throughout the food chain from the fishing vessel or aquaculture farm to the consumer's table. Although seafood labelling has to include the commercial designation, scientific name, geographical area, production method and state whether the product has been previously frozen, the commercial fish species available on the market cannot always be easily identified in processed and prepared fishery products, especially when morphological features have been removed.

Therefore, the current importance of the fish trade requires technological developments in food production, handling, processing and distribution by a global network of operators in order to guarantee the authenticity and the origin of fish and seafood products (Gil, 2007; Rasmussen and Morrissey, 2008; Di Pinto et al., 2013).

The results of this study reveal a high occurrence of incorrect species declaration in prepared fish fillet products, further evidence of the need for increased traceability and assessment of the authenticity of food products. In fact, cases of fraudulent mislabelling of lesservalued species are becoming more common as commercial quotas on certain high-value species become more restrictive in the world
(Barbuto et al., 2010; Miller and Mariani, 2010; Cawthorn et al., 2011).

Fishery products substitution of valuable species of lower value is common practice because it is easy with a immediate economic reward. Generally, the species used in substitution have different and lower nutritional value compared with those declared as showed in this study. In addition, the substitution may be favored by the depletion in some areas of highly appreciated species, the high variety of fish species, the difficult differential diagnosis and the overall lack of taxonomical expertise. Moreover, fish identification may be insufficient if there are overlapping features between taxa, as it frequently occurs in many fish species (Di Pinto et al., 2015).

This study highlights the need for the sustainable management of aquatic resources, in particular, showed widespread use of species of lower commercial value and from highly polluted waters of African countries such as Pangasius hypophthalmus and Merluccius capensis respectively (Filonzi et al., 2010). Given the increase in consumption and production of convenience ready-to-cook seafood, precautionary measures are necessary. Traceability is an essential component of any risk management strategy, and a key requirement for post-marketing surveillance. The fishing industry requires a full traceability system, a crucial step in promoting greater seafood safety, quality and sustainability (Di Pinto et al., 2015).

\section{Conclusions}

Given the increasing demand for transparency in the food industry, the enforcement of proper labelling have provided a driving force for the development of suitable analytical methodologies for species identification. Indeed, the seafood industry currently lacks a simple, standardized, widespread method for tracing seafood products purchased along the supply chain. Specifically, DNA traceability could offer a more precise form of traceability for fish and byproducts, as provided by Council Regulation (EC) No 1224/2009, art. 13 (European Commission, 2009). A tracing system that combines genetic analysis with conventional methods of traceability may give food companies and consumers the information they need to make sustainable seafood choices. A great effort should therefore be made to create a strong standardized monitoring program or strategy and to evoke consumer awareness on several aspects of accurate labelling information (Di Pinto et al., 2015). 
Table 1. Sole, plaice, hake and Atlantic salmon fillets results.

\begin{tabular}{|c|c|c|c|c|c|c|c|c|}
\hline $\begin{array}{l}\text { Sample } \\
\text { number }\end{array}$ & $\begin{array}{l}\text { Common } \\
\text { name }^{\circ}\end{array}$ & $\begin{array}{l}\text { Latin } \\
\text { name }^{\circ}\end{array}$ & $\begin{array}{l}\text { Catch } \\
\text { location }\end{array}$ & $\begin{array}{l}\text { Similarity } \\
(\%)\end{array}$ & $\begin{array}{l}\text { True } \\
\text { common name }^{\circ}\end{array}$ & $\begin{array}{c}\text { True } \\
\text { Latin name }^{\circ} \text { (COI ID.) }\end{array}$ & Genbank A.N. & Mislabelling \\
\hline 1 & Sole & n.a & n.a & 99 & Sole & Solea solea & EU513746.1 & No \\
\hline 2 & Sole & Solea solea & FAO 37 & 99 & Sole & Solea solea & EU513746.1 & No \\
\hline 3 & Sole & n.a & n.a & 100 & Atlantic sole & Solea senegalensis & KF3691186.1 & Yes \\
\hline 4 & Sole & n.a & FAO 37 & 99 & Sole & Solea solea & EU513746.1 & No \\
\hline 5 & Sole & n.a & n.a & 99 & Atlantic sole & Solea senegalensis & KF3691186.1 & Yes \\
\hline 6 & Sole & Solea solea & FAO 27 & 99 & Atlantic sole & Solea senegalensis & KF3691186.1 & Yes \\
\hline 7 & Sole & n.a & FAO 27 & 99 & Sole & Solea solea & EU513746.1 & No \\
\hline 8 & Sole & n.a & FAO 27 & 99 & Atlantic sole & Solea senegalensis & KF3691186.1 & Yes \\
\hline 9 & Sole & n.a & FAO 37 & 99 & Sole & Solea solea & EU513746.1 & No \\
\hline 10 & Sole & Solea solea & n.a & 99 & Sole & Solea solea & EU513746.1 & No \\
\hline 11 & Sole & n.a & n.a & 100 & Atlantic sole & Solea senegalensis & KF3691186.1 & Yes \\
\hline 12 & Sole & n.a & FAO 27 & 99 & Atlantic sole & Solea senegalensis & KF3691186.1 & Yes \\
\hline 13 & Sole & Solea solea & n.a & 99 & Sole & Solea solea & EU513746.1 & No \\
\hline 14 & Sole & Solea solea & n.a & 99 & Sole & Solea solea & EU513746.1 & No \\
\hline 15 & Sole & n.a & n.a & 99 & Atlantic sole & Solea senegalensis & KF3691186.1 & Yes \\
\hline 16 & Sole & Solea solea & FAO 27 & 99 & Sole & Solea solea & EU513746.1 & No \\
\hline 17 & Sole & n.a & n.a & 99 & Atlantic sole & Solea senegalensis & KF3691186.1 & Yes \\
\hline 18 & Sole & Solea solea & n.a & 99 & Sole & Solea solea & EU513746.1 & No \\
\hline 19 & Sole & n.a & n.a & 99 & Atlantic sole & Solea senegalensis & KF3691186.1 & Yes \\
\hline 20 & Sole & Solea solea & FAO 27 & 99 & Sole & Solea solea & EU513746.1 & No \\
\hline 21 & Sole & Solea solea & FAO 27 & 99 & Sole & Solea solea & EU513746.1 & No \\
\hline 22 & Sole & n.a & FAO 37 & 99 & Sole & Solea solea & EU513746.1 & No \\
\hline 23 & Sole & n.a & n.a & 100 & Atlantic sole & Solea senegalensis & KF3691186.1 & Yes \\
\hline 24 & Sole & Solea solea & FAO 27 & 99 & Atlantic sole & Solea senegalensis & KF3691186.1 & Yes \\
\hline 25 & Sole & Solea solea & FAO 37 & 99 & Sole & Solea solea & EU513746.1 & No \\
\hline 26 & Sole & Solea solea & n.a & 99 & Sole & Solea solea & EU513746.1 & No \\
\hline 27 & Sole & n.a & FAO 27 & 99 & Atlantic sole & Solea senegalensis & KF3691186.1 & Yes \\
\hline 28 & Plaice & n.a & FAO 27 & 100 & Plaice & Pleuronectes platessa & EU513682.1 & No \\
\hline 29 & Plaice & n.a & FAO 27 & 99 & Plaice & Pleuronectes platessa & EU513682.1 & No \\
\hline 30 & \multicolumn{2}{|c|}{ Plaice Pleuronectes platessa } & FAO 27 & 99 & Pangasius & Pangasius hypophtalmus & JF292402.1 & Yes \\
\hline 31 & Plaice & n.a & FAO 27 & 99 & Pangasius & Pangasius hypophtalmus & JF292402.1 & Yes \\
\hline 32 & \multicolumn{2}{|c|}{ Plaice Pleuronectes platessa } & FAO 27 & 99 & Pangasius & Pangasius hypophtalmus & JF292402.1 & Yes \\
\hline 33 & \multicolumn{2}{|c|}{ Plaice Pleuronectes platessa } & FAO 27 & 99 & Plaice & Pleuronectes platessa & EU513682.1 & No \\
\hline 34 & \multicolumn{2}{|c|}{ Plaice Pleuronectes platessa } & FAO 27 & 98 & Plaice & Pleuronectes platessa & EU513682.1 & No \\
\hline 35 & Plaice & n.a & FAO 27 & 98 & Pangasius & Pangasius hypophtalmus & JF292402.1 & Yes \\
\hline 36 & \multicolumn{2}{|c|}{ Plaice Pleuronectes platessa } & FAO 27 & 99 & Plaice & Pleuronectes platessa & EU513682.1 & No \\
\hline 37 & \multicolumn{2}{|c|}{ Plaice Pleuronectes platessa } & FAO 27 & 100 & Plaice & Pleuronectes platessa & EU513682.1 & No \\
\hline 38 & \multicolumn{2}{|c|}{ Plaice Pleuronectes platessa } & n.a & 99 & Pangasius & Pangasius hypophtalmus & JF292402.1 & Yes \\
\hline 39 & \multicolumn{2}{|c|}{ Plaice Pleuronectes platessa } & $\mathrm{FAO} 27$ & 99 & Plaice & Pleuronectes platessa & EU513682.1 & No \\
\hline 40 & \multicolumn{2}{|c|}{ Plaice Pleuronectes platessa } & FAO 27 & 99 & Plaice & Pleuronectes platessa & EU513682.1 & No \\
\hline 41 & \multicolumn{2}{|c|}{ Plaice Pleuronectes platessa } & FAO 27 & 99 & Plaice & Pleuronectes platessa & EU513682.1 & No \\
\hline 42 & Plaice & n.a & FAO 27 & 99 & Pangasius & Pangasius hypophtalmus & JF292402.1 & Yes \\
\hline 43 & Plaice & n.a & FAO 27 & 99 & Plaice & Pleuronectes platessa & EU513682.1 & No \\
\hline 44 & Plaice & n.a & FAO 27 & 99 & Pangasius & Pangasius hypophtalmus & JF292402.1 & Yes \\
\hline 45 & \multicolumn{2}{|c|}{ Plaice Pleuronectes platessa } & FAO 27 & 99 & Pangasius & Pangasius hypophtalmus & JF292402.1 & Yes \\
\hline 46 & \multicolumn{2}{|c|}{ Plaice Pleuronectes platessa } & FAO 27 & 99 & Plaice & Pleuronectes platessa & EU513682.1 & No \\
\hline 47 & \multicolumn{2}{|c|}{ Plaice Pleuronectes platessa } & FAO 71 & 99 & Plaice & Pleuronectes platessa & EU513682.1 & No \\
\hline 48 & Plaice & n.a & FAO 27 & 99 & Pangasius & Pangasius hypophtalmus & JF292402.1 & Yes \\
\hline 49 & \multicolumn{2}{|c|}{ Plaice Pleuronectes platessa } & FAO 27 & 100 & Plaice & Pleuronectes platessa & EU513682.1 & No \\
\hline 50 & Plaice & n.a & n.a & 99 & Pangasius & Pangasius hypophtalmus & JF292402.1 & Yes \\
\hline
\end{tabular}


Table 1. Continued from previous page.

\begin{tabular}{|c|c|c|c|c|c|c|c|c|}
\hline $\begin{array}{l}\text { Sample } \\
\text { number }\end{array}$ & $\begin{array}{c}\text { Common } \\
\text { name }^{\circ}\end{array}$ & $\begin{array}{l}\text { Latin } \\
\text { name }^{\circ}\end{array}$ & $\begin{array}{l}\text { Catch } \\
\text { location }\end{array}$ & $\begin{array}{c}\text { Similarity } \\
(\%)\end{array}$ & $\begin{array}{l}\text { True } \\
\text { common } \\
\text { name }^{\circ}\end{array}$ & $\begin{array}{c}\text { True } \\
\text { Latin } \\
\text { name }^{\circ}(\mathrm{COI} \text { ID. })\end{array}$ & Genbank A.N. & Mislabelling \\
\hline 51 & Plaice & n.a & n.a & 99 & Pangasius & Pangasius hypophtalmus & JF292402.1 & Yes \\
\hline 52 & Plaice & n.a & FAO 27 & 99 & Pangasius & Pangasius hypophtalmus & JF292402.1 & Yes \\
\hline 53 & Plaice & Pleuronectes platessa & FAO 27 & 99 & Plaice & Pleuronectes platessa & EU513682.1 & No \\
\hline 54 & Plaice & n.a & FAO 27 & 99 & Plaice & Pleuronectes platessa & EU513682.1 & No \\
\hline 55 & Plaice & Pleuronectes platessa & n.a & 99 & Pangasius & Pangasius hypophtalmus & JF292402.1 & Yes \\
\hline 56 & Atlantic salmon & Salmo salar & FAO 27 & 99 & Atlantic salmon & Salmo salar & FJ399413.1 & No \\
\hline 57 & Atlantic salmon & n.a & n.a & 99 & Atlantic salmon & Salmo salar & FJ399413.1 & No \\
\hline 58 & Atlantic salmon & Salmo salar & n.a & 99 & Atlantic salmon & Salmo salar & FJ399413.1 & No \\
\hline 59 & Atlantic salmon & Salmo salar & FAO 27 & 99 & Atlantic salmon & Salmo salar & FJ399413.1 & No \\
\hline 60 & Atlantic salmon & Salmo salar & FAO 27 & 99 & Atlantic salmon & Salmo salar & FJ399413.1 & No \\
\hline 61 & Atlantic salmon & n.a & FAO 27 & 99 & Atlantic salmon & Salmo salar & FJ399413.1 & No \\
\hline 62 & Atlantic salmon & Salmo salar & FAO 27 & 99 & Atlantic salmon & Salmo salar & FJ399413.1 & No \\
\hline 63 & Atlantic salmon & Salmo salar & n.a & 99 & Atlantic salmon & Salmo salar & FJ399413.1 & No \\
\hline 64 & Atlantic salmon & n.a & FAO 27 & 100 & Atlantic salmon & Salmo salar & FJ399413.1 & No \\
\hline 65 & Atlantic salmon & Salmo salar & FAO 27 & 99 & Atlantic salmon & Salmo salar & FJ399413.1 & No \\
\hline 66 & Atlantic salmon & Salmo salar & FAO 27 & 99 & Atlantic salmon & Salmo salar & FJ399413.1 & No \\
\hline 67 & Atlantic salmon & n.a & n.a & 99 & Atlantic salmon & Salmo salar & FJ399413.1 & No \\
\hline 68 & Atlantic salmon & Salmo salar & n.a & 99 & Atlantic salmon & Salmo salar & FJ399413.1 & No \\
\hline 69 & Hake & Merluccius merluccius & FAO 37 & 98 & Hake & Merluccius merluccius & FJ460768.1 & No \\
\hline 70 & Hake & Merluccius merluccius & FAO 37 & 99 & Hake & Merluccius merluccius & FJ460768.1 & No \\
\hline 71 & Hake & n.a & n.a & 99 & Atlantic hake & Merluccius hubbsi & EU074472.1 & Yes \\
\hline 72 & Hake & Merluccius merluccius & n.a & 99 & Atlantic hake & Merluccius hubbsi & EU074472.1 & Yes \\
\hline 73 & Hake & Merluccius merluccius & FAO 37 & 99 & Hake & Merluccius merluccius & FJ460768.1 & No \\
\hline 74 & Hake & n.a & n.a & 98 & Pacific hake & Merluccius productus & FJ164843.1 & Yes \\
\hline 75 & Hake & Merluccius merluccius & n.a & 99 & Hake & Merluccius merluccius & FJ460768.1 & No \\
\hline 76 & Hake & Merluccius merluccius & FAO 37 & 100 & Hake & Merluccius merluccius & FJ460768.1 & No \\
\hline 77 & Hake & Merluccius merluccius & FAO 37 & 99 & Hake & Merluccius merluccius & FJ460768.1 & No \\
\hline 78 & Hake & n.a & FAO 37 & 99 & Atlantic hake & Merluccius hubbsi & EU074472.1 & Yes \\
\hline 79 & Hake & n.a & n.a & 98 & Pacific hake & Merluccius productus & FJ164843.1 & Yes \\
\hline 80 & Hake & n.a & n.a & 99 & Pacific hake & Merluccius productus & FJ164843.1 & Yes \\
\hline 81 & Hake & Merluccius merluccius & FAO 37 & 100 & Hake & Merluccius merluccius & FJ460768.1 & No \\
\hline 82 & Hake & n.a & n.a & 99 & South African hake & Merluccius capensis & JF493884.1 & Yes \\
\hline 83 & Hake & Merluccius merluccius & FAO 37 & 99 & Hake & Merluccius merluccius & FJ460768.1 & No \\
\hline 84 & Hake & Merluccius merluccius & FAO 37 & 99 & Hake & Merluccius merluccius & FJ460768.1 & No \\
\hline 85 & Hake & n.a & n.a & 99 & South African hake & Merluccius capensis & JF493884.1 & Yes \\
\hline 86 & Hake & n.a & FAO 37 & 99 & Hake & Merluccius merluccius & FJ460768.1 & No \\
\hline 87 & Hake & n.a & n.a & 99 & Hake & Merluccius merluccius & FJ460768.1 & No \\
\hline 88 & Hake & Merluccius merluccius & FAO 37 & 99 & Hake & Merluccius merluccius & FJ460768.1 & No \\
\hline 89 & Hake & n.a & n.a & 99 & Pacific hake & Merluccius productus & FJ164843.1 & Yes \\
\hline 90 & Hake & n.a & FAO 37 & 100 & Atlantic hake & Merluccius hubbsi & EU074472.1 & Yes \\
\hline 91 & Hake & n.a & n.a & 99 & Atlantic hake & Merluccius hubbsi & EU074472.1 & Yes \\
\hline 92 & Hake & n.a & n.a & 99 & Atlantic hake & Merluccius hubbsi & EU074472.1 & Yes \\
\hline 93 & Hake & Merluccius merluccius & FAO 37 & 99 & Hake & Merluccius merluccius & FJ460768.1 & No \\
\hline 94 & Hake & n.a & n.a & 99 & South African hake & Merluccius capensis & JF493884.1 & Yes \\
\hline 95 & Hake & n.a & n.a & 99 & South African hake & Merluccius capensis & JF493884.1 & Yes \\
\hline 96 & Hake & n.a & n.a & 99 & Atlantic hake & Merluccius hubbsi & EU074472.1 & Yes \\
\hline 97 & Hake & n.a & n.a & 99 & Atlantic hake & Merluccius hubbsi & EU074472.1 & Yes \\
\hline 98 & Hake & n.a & n.a & 98 & Pacific hake & Merluccius productus & FJ164843.1 & Yes \\
\hline
\end{tabular}

n.a, not available. ${ }^{\circ}$ Latin and common name according to MiPAAF (2008). 
http://www.geneious.com/

References

Armani A, Guardone L, La Castellana R, Gianfaldoni D, Guidi A, Castigliego L, 2015. DNA barcoding reveals commercial and health issues in ethnic seafood sold on the Italian market. Food Control 55:20614.

Barbuto M, Galimberti A, Ferri E, Labra M, Malandra R, Galli P, Casiraghi M, 2010. DNA barcoding reveals fraudulent substitutions in shark seafood products: the Italian case of "Palombo" (Mustelus spp.). Food Res Int 43:376-81.

Bottero MT, Dalmasso A, 2011. Animal species identification in food products: evolution of biomolecular methods. Vet J 190:34-8.

Cawthorn DM, Steinman HA, Witthuhn RC, 2011. DNA barcoding reveals a high incidence of fish species misrepresentation and substitution on the South African market. Food Res Int 46:30-40.

Cline E, 2012. Marketplace substitution of Atlantic salmon for Pacific salmon in Washington State detected by DNA barcoding. Food Res Int 45:388-93.

Di Pinto A, Di Pinto P, Terio V, Bozzo G, Bonerba E, Ceci E, Tantillo G, 2013. DNA barcoding for detecting market substitution in salted cod fillets and battered cod chunks. Food Chem 141:1757-62.

Di Pinto A, Marchetti P, Mottola A, Bozzo G, Bonerba E, Ceci E, Bottaro M, Tantillo G, 2015. Species identification in fish fillet products using DNA barcoding. Fish Res 170:9-13.

Di Pinto A, Mottola A, Marchetti P, Bottaro M, Terio V, Bozzo G, Bonerba E, Ceci E, Tantillo G. Packaged frozen fishery products species identification, mislabeling occurrence and legislative implications. Food Chem (in press).

Drummond AJ, Ashton B, Buxton S, Cheung M, Cooper A, Duran C, Field M, Heled J, Kearse M, Markowitz S, Moir R, StonesHavas S, Sturrock S, Thierer T, Wilson A, 2011. Geneious v5.4. Available from:
European Commission, 2009. Council Regulation of 20 November 2009 establishing a Community control system for ensuring compliance with the rules of the common fisheries policy, amending Regulations (EC) No 847/96, (EC) No 2371/2002, (EC) No 811/2004, (EC) No $768 / 2005$, (EC) No 2115/2005, (EC) No 2166/2005, (EC) No 388/2006, (EC) No 509/2007, (EC) No 676/2007, (EC) No 1098/2007, (EC) No 1300/2008, (EC) No $1342 / 2008$ and repealing Regulations (EEC) No 2847/93, (EC) No 1627/94 and (EC) No 1966/2006, 1224/2009/EC. In: Official Journal, L 343, 22-12-2009.

European Commission, 2013. Regulation of the European Parliament and of the Council of 11 December 2013 on the common organisation of the markets in fishery and aquaculture products, amending Council Regulations (EC) No 1184/2006 and (EC) No 1224/2009 and repealing Council Regulation (EC) No 104/2000, 1379/2013/EU. In: Official Journal, L 354, 28.12.2013.

Filonzi L, Chiesa S, Vaghi M, Nonnis Marzano F, 2010. Molecular barcoding reveals mislabelling of commercial fish products in Italy. Food Res Int 43:1383-8.

Garcia-Vazquez E, Perez J, Martinez JL, Pardiñas AF, Lopez B, Karajskou N, Casa MF, Machado-Schiaffino G, Triantafyllidis A, 2011. High level of mislabelling in Spanish and Greek hake markets suggests the fraudulent introduction of African species. J Agr Food Chem 59:475-80.

Gil LA, 2007. PCR-based methods for fish and fishery products authentication. Trends Food Sci Tech 18:558-66.

Handy SM, Deeds JR, Ivanova NV, Hebert PDN, Hanner RH, Ormos A, Weigt LA, Moore MM, Yancy HF, 2011. A single-laboratory validated method for the generation of DNA barcodes for the identification of fish for regulatory compliance. J AOAC Int 94:1-10.

Hanner R, Becker S, Ivanova NV, Steinke D,
2011. FISH-BOL and seafood identification: geographically dispersed case studies reveal systemic market substitution across Canada. Mit DNA Suppl 1:106-22.

Hebert PD, Ratnasingham S, de Waard JR, 2003. Barcoding animal life: cytochrome $\mathrm{c}$ oxidase subunit 1 divergences among closely related species. P Roy Soc B-Biol Sci 1:96-9.

Holmes BH, Steinke D, Ward RD, 2009. Identification of shark and ray fins using DNA barcoding. Fish Res 95:280-8.

Jacquet JL, Pauly D, 2008. Trade secrets: renaming and mislabelling of seafood. Mar Policy 32:309-18.

Marko PB, Lee SC, Rice AM, Gramling JM, Fitzhenry TM, McAlister JS, Harper GR, Moran AL, 2004. Mislabelling of a depleted reef fish. Nature 430:309-10.

Miller D, Jessel A, Mariani S, 2012. Seafood mislabelling: comparisons of two western European case studies assist in defining influencing factors, mechanisms and motives. Fish Fish 13:345-8.

Miller D, Mariani S, 2010. Smoke mirrors and mislabelled cod: poor transparency in the European seafood industry. Front Ecol Environ 8:517-21.

MiPAAF, 2008. Decree of the Italian Ministry of Agricultural, Food and Forestry Policies of 31 January 2008. Italian name for fish species of commercial interest. Available from: https://www.politicheagricole. it/flex/cm/pages/ServeBLOB.php/L/TT/IDPa gina/2563

Rasmussen RS, Morrissey MT, 2008. DNAbased methods for the identification of commercial fish and seafood species. Compr Rev Food Sci F 7:280-95.

Ward RD, Holmes BH, White WT, Last PR, 2008. DNA barcoding, Australasian chondrichthyans: results and potential uses in conservation. Mar Fresh Res 59:57-71.

Wong EHK, Hanner RH, 2008. DNA barcoding detects market substitution in North American seafood. Food Res 41:828-37. 